\title{
Health Literacy in Individuals at Risk for Alzheimer's Dementia: A Systematic Review
}

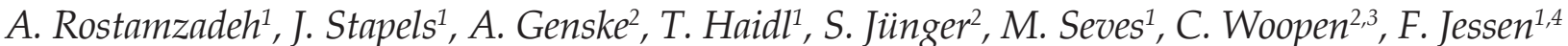

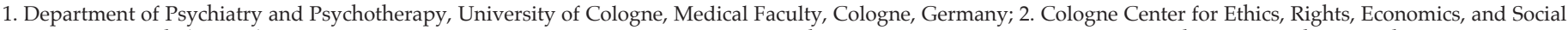

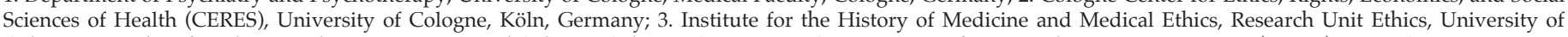
Cologne, Faculty of Medicine and University Hospital Cologne, Cologne, Germany; 4. German Center for Neurodegenerative Diseases (DZNE), Bonn, Germany

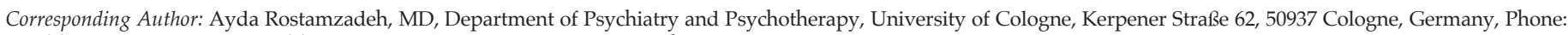
+49 (0)221 - 478 3870, Fax: +49 (0)221 - 478 6030, E-Mail: ayda.rostamzadeh@uk-koeln.de

\begin{abstract}
BACKGROUND: Health literacy (HL) refers to the capacity to access, understand, appraise and apply information for decision-making and acting in health-related matters. In the field of Alzheimer's disease (AD), expanding technologies of early disease detection, disease course prediction and eventually personalized prevention confront individuals at-risk with increasingly complex information, which demand substantial HL skills. Here we report current findings of HL research in at-risk groups.

METHODS: Search strings, referring to HL, AD, amyloid and risk, were developed. A systematic review was conducted in PUBMED, Cochrane Library, PsycINFO, and Web of Science to summarize the state of evidence on HL in at-risk individuals for Alzheimer's dementia. Eligible articles needed to employ a validated tool for HL, mention the concept or one dimension (access, understand, appraise and apply information for decision-making and acting).

RESULTS: 26 quantitative and 9 qualitative studies addressing at least one dimension of HL were included. Overall, there is evidence for a wish to gain knowledge about the own brain status and risk of dementia. Psychological distress may occur and the subjective benefit-risk estimation may be modified after risk disclosure. Effects on lifestyle and planning may occur. Overall understanding and appraisal of information related to AD risk seem variable with several impacting factors. In mild cognitive impairment (MCI) basic HL skill seem to be affected by cognitive dysfunction.

CONCLUSIONS: Systematic assessment of HL in at-risk population for AD is sparse. Findings indicate the paramount importance of adequate communication with persons at risk, being sensitive to individual needs and preferences. Substantial research needs were identified.
\end{abstract}

Key words: Health literacy, individuals at risk, access, understanding and evaluating health information, decision making in health, mild cognitive impairment, Alzheimer's disease.

\section{Introduction}

\section{Current state of research in early detection of Alzheimer's disease}

A lzheimer's disease (AD), as the underlying cause of Alzheimer's dementia, has become a major public health challenge. The pathophysiological processes of AD start decades before its symptom onset and can be identified early in the course of the disease by biomarkers of amyloid and tau deposition as well as of neurodegeneration (1). Current research criteria allow biomarker-based diagnosis in the prodromal and even in the asymptomatic stage, long before functional disability of dementia becomes apparent (1-3). This has stimulated extensive research on early disease identification, dementia risk-prediction and prevention strategies in $\mathrm{AD}$ with the ultimate aim of slowing the disease course by impacting on modifiable risk factors in lifestyle-based interventions (4) and targeting molecular pathways of $\mathrm{AD}$ by pharmacological approaches (5-7). In all such cases, interventions start at a pre-dementia stage and focus on selected groups of at-risk individuals.

\section{Health Literacy in at-risk groups for Alzheimer's dementia}

Health literacy (HL) is a new concept which can be described as the specific knowledge, competency and skills with respect to health-related matters (8). Sørensen et al. (9) integrated existing concepts and describe HL as a person's ability to (1) access, (2) understand and (3) appraise medical or clinical issues and (4) apply health information. As HL reflects these multidimensional abilities and skills, it is believed to play an essential role in the design and eventually success of selective and targeted prevention programs. In the case of $\mathrm{AD}$, selective prevention addresses healthy individuals with an increased risk for $\mathrm{AD}$, such as healthy carriers of the 
risk-enhancing Apolipoprotein E4 (APOE4) genotype, while targeted prevention in $\mathrm{AD}$ aims at symptomatic individuals with subjective cognitive decline (SCD) or mild cognitive impairment $(\mathrm{MCI})(2,3)$. To date, knowledge about HL in individuals with very early $\mathrm{AD}$ and increased risk of dementia is very limited, which significantly limits the development of adequate communication approaches for research and clinical practice. Taking into account that the longitudinal deterioration of cognitive functioning in these patients will affect HL skills progressively, there are unique challenges concerning counselling and informed consent procedures, but also public health campaigns. In addition, there is little knowledge on the overall engagement of such individuals in the health-care system.

This is, to the best of our knowledge, the first review systematically investigating the current state of knowledge about HL in at-risk individuals for Alzheimer's dementia. The goal of this review is to provide a summary of the evidence on how at-risk groups for Alzheimer's dementia gain access to, understand, appraise and apply risk-related health information in decision-making and acting. We focus specifically on studies which employed biomarkers of amyloid pathology or genetic risk factors.

\section{Methods}

\section{Conceptual framework}

This review is based on the integrative model of HL developed by the HLS-EU Consortium in 2012, and on the Australian HL concept outlined in the Health Literacy National Statement (AHLNS) (10).

Using the definition by Sørensen et al. (9) we developed a search strategy that covers HL as an umbrella term as well as its four subdomains (access, understand, appraise, apply). For the purpose of this review, we decided to split the step "apply" into two sub categories: decision-making and action; this allows us to better understand gaps between the phases of intention (decision-making) and actual health behavior change (action).

\section{Search strategy}

Search strings consisted of three sections that were combined using the Boolean Operator "AND". One section was referring to $\mathrm{HL}$, one was the term "Alzheimer's disease" and "amyloid" and one was "risk" and "risk factors" (see appendix 1 for the detailed search strings). The final search was carried out in PUBMED, Cochrane Library, PsycINFO and Web of Science. The period of literature search was between March 2017 and March 2018 (last read out 21.03.2018). Furthermore, the reference lists of all publications included in this review were hand searched for additional studies. Search strategy, screening and data selection were carried out in accordance with the PRISMA criteria (11). This review is registered in the international prospective register of systematic reviews (PROSPERO) with the registration number: CRD42016052345 (http:/ / www.crd.york.ac.uk/ PROSPERO/display_record.php?ID=CRD42016052345). The date of registration was 12.12.2016.

\section{Paper selection/Inclusion criteria}

We included studies that investigated individuals at risk of developing Alzheimer's dementia. These were individuals with SCD or MCI and those with a family history of $\mathrm{AD}$ or with biological risk, such as a genetic predisposition (i.e. healthy siblings of individuals with monogenic early-onset AD (EOAD) or carriers of the APOE4 allele) or individuals with evidence for amyloidpathology measured by biomarkers. To be included in this review, articles had to be published in a peerreviewed journal and written in English or German. No starting time point regarding the publication date was applied. This review considered all types of study designs including quantitative (such as observational, prospective and retrospective cohort studies, clinical trials), qualitative and mixed methods designs. To be included, studies had to use a validated tool to measure HL or examine any of its dimensions (access, understanding, appraisal, decision-making or action) as a primary or secondary outcome. Alternatively, studies had to mention the concept of HL plus at least one of the four dimensions in the title or abstract.

\section{Screening and Assessment}

Articles were screened for eligibility based on title and abstract by two independent reviewers and then checked independently according to the inclusion criteria. Any discrepancies in rating were resolved through discussion, and when necessary, a third reviewer judged the respective publication. If eligibility was unclear, but studies deemed potentially relevant by title or abstract, a full text review was performed as in all articles selected for full text review.

\section{Data extraction}

Due to the heterogeneity of study designs and outcomes, we decided to conduct a narrative review. The complex nature of the research question made it necessary to include a variety of study types including different quantitative formats as well as qualitative interview studies. Therefore, we applied the methodology of a mixed-methods review. For data extraction and synthesis, we followed recommendations for appraising evidence from different study types 
within one review $(12,13)$. In order to ensure adequate data extraction, an evaluation matrix for data analyses was designed based on the inclusion criteria and our research question (see appendix 2). Along this form, study characteristics (author, title, journal, year of publication and country of origin) were extracted. The next steps included extraction of additional information on study design, characteristics and population and regarding the main outcome measures. We applied a segregated methodology (14), where we first analyzed and synthesized data from quantitative studies, before we examined qualitative findings in a separate step. In the final step, all findings were combined in a narrative synthesis. The narrative includes the target population characteristics, the HL process, the methodology, the study setting, and the type of outcome. Thematic categories were predefined based on the research question and were further refined during the data analysis process. Data analyses was performed by two independent reviewers and in case of any discrepancies, a third reviewer judged re-evaluated.

\section{Quality assessment (Risk of bias)}

The quality of included studies was evaluated by two independent reviewers using a standardized set of criteria proposed by Hawker et al. in 2002 for mixedmethods reviews (see appendix 3) (15). Discrepancies between raters were resolved by discussion and where necessary re-assessed by a third reviewer.

\section{Results}

\section{Included Studies}

The initial search yielded 7804 papers. 112 articles were identified through reference check. 43 articles were selected for full text review. After full text review, 35 studies fulfilled the inclusion criteria for analysis. The detailed selection process is depicted in figure 1.

The 35 papers meeting the inclusion criteria are summarized in appendix 4 . A total of 27 studies were carried out in the USA $(16,17,26-35,18,36-42,19-25)$, two in Austria $(43,44)$ and one in each of the following countries: Cuba (45), the Netherlands (46), Germany (47), United Kingdom (48), Belgium (49) and Sweden (50). We neither found studies that considered HL as a basic concept for the particular investigation, nor studies using established assessment tools for HL, such as HLS-EU, HLQ, REALM or TOFLHA. However, we identified studies where at least one aspect of HL was actively applied. The majority of identified studies used customized tools for their individual study purposes to measure single domains of HL. Four studies used established measures for single domains of HL, such as the health numeracy scale $(24,43)$ and the tool for
Capacity to Consent to Treatment, an instrument to evaluate medical-decision making capacity (30, 31). Regarding the target populations, we identified reports with symptomatic individuals such as MCI patients (9 studies) and patients with SCD (1 study). Furthermore, we identified studies on healthy adults with first-degree relatives suffering from Alzheimer's dementia (21 studies) and healthy first-degree relatives of individuals with monogenetic early onset AD EOAD (4 studies). Three studies investigated disclosure on brain amyloid-status in individuals with MCI and SCD (40, $41,49)$. We included one qualitative study addressing mild Alzheimer's dementia patients and their relatives, because we considered the results of the interviews important regarding the patients' decision-making capacities, self-determination and autonomy (47). A total of 20 studies were carried out as part of the REVEALprogram (Risk Evaluation and Education for AD), which is a series of multi-site randomized controlled clinical trials with the objective to evaluate the impact of genetic risk assessment and disclosure in healthy adults with first-degree relatives with Alzheimer's dementia $(35,38)$.

According to the quality assessment a total of 11 publications were rated as "good," 21 as "fair" and 3 papers as "poor" (see appendix 3). The articles rated as "poor" were not excluded from the study, because the findings were considered as relevant for our research question, but need to be evaluated with consideration due to the quality rating.

\section{Findings for the different HL domains}

As highlighted above, individuals at risk for Alzheimer's dementia can be grouped into asymptomatic individuals, such as healthy adults with first-degree relatives with Alzheimer's dementia, and into symptomatic individuals, including MCI and SCD patients. Therefore, we decided to present the results of the two target populations separately. The thematic fields of the findings within the different HL domains are summarized in table 1 .

\section{Access to health-related information on $A D$}

\section{Access in cognitively impaired individuals}

Access to health-related information in cognitively impaired individuals has not been investigated in the included studies.

\section{Access in cognitively unimpaired individuals}

Two studies reported that first-degree relatives of $\mathrm{AD}$ patients obtained and accessed health-related information about AD mainly informally through spouses, friends, literature, patient organizations and mass media and less likely obtained disease-related information through health care providers $(20,50)$. 
Table 1. Content analysis within the different HL domains, clustered in cognitively impaired and unimpaired individuals

\begin{tabular}{|c|c|c|c|c|}
\hline \multicolumn{4}{|c|}{ Content analysis within the different HL domains } & \multirow[b]{2}{*}{$\begin{array}{l}\text { Apply: Effects on health behavior (health } \\
\text { behavior change) }\end{array}$} \\
\hline Access & Understand & Appraise & Apply: Decision-Making & \\
\hline Cognitively impaired & Cognitively impaired & Cognitively impaired & Cognitively impaired & Cognitively impaired \\
\hline No data & $\begin{array}{l}\text { - medical decision-making capacity } \\
\text { - health numeracy } \\
\text { - risk disclosure of AD biomarker } \\
\text { test results } \\
\text { - improvement of risk disclosure } \\
\text { comprehension }\end{array}$ & $\begin{array}{l}\text { - framing effects } \\
\text { - experiences and coping mechanisms }\end{array}$ & $\begin{array}{l}\text { - decision to undergo actual or hypo- } \\
\text { thetical biomarker testing for AD } \\
\text { - decision to participate in a hypothe- } \\
\text { tical trial with a disease-modifying } \\
\text { treatment for AD }\end{array}$ & - lifestyle changes \\
\hline Cognitively unimpaired & Cognitively unimpaired & Cognitively unimpaired & Cognitively unimpaired & Cognitively unimpaired \\
\hline $\begin{array}{l}\text { - access health-related } \\
\text { AD information } \\
\text { - access to information } \\
\text { on genetic APOE-tes- } \\
\text { ting }\end{array}$ & $\begin{array}{l}\text { - general knowledge about AD } \\
\text { - information needs } \\
\text { - recall of disclosed Alzheimer's de- } \\
\text { mentia risk in APOE genetic testing } \\
\text { - ethnic differences regarding } \\
\text { knowledge in APOE genotyping }\end{array}$ & $\begin{array}{l}\text { - perceived risk of developing } \mathrm{AD} \text {, } \\
\text { psychosocial effects and influencing } \\
\text { factors on risk perception } \\
\text { - Experiences and coping mechanisms } \\
\text { - perceptions of benefits and risks of } \\
\text { genetic APOE-testing }\end{array}$ & $\begin{array}{l}\text { - intentions / motivation to get tested/ } \\
\text { to undergo genetic APOE-testing } \\
\text { - intentions/decisions to change } \\
\text { health behavior } \\
\text { - intentions/motivation to get tested/ } \\
\text { to undergo genetic APOE-testing } \\
\text { - intentions/decisions to change } \\
\text { health behavior }\end{array}$ & $\begin{array}{l}\text { - motivation for having participated in } \\
\text { presymptomatic AD testing } \\
\text { - lifestyle changes } \\
\text { - psychosocial effects }\end{array}$ \\
\hline
\end{tabular}

\section{Figure 1. PRISMA Flow-Chart of paper selection}

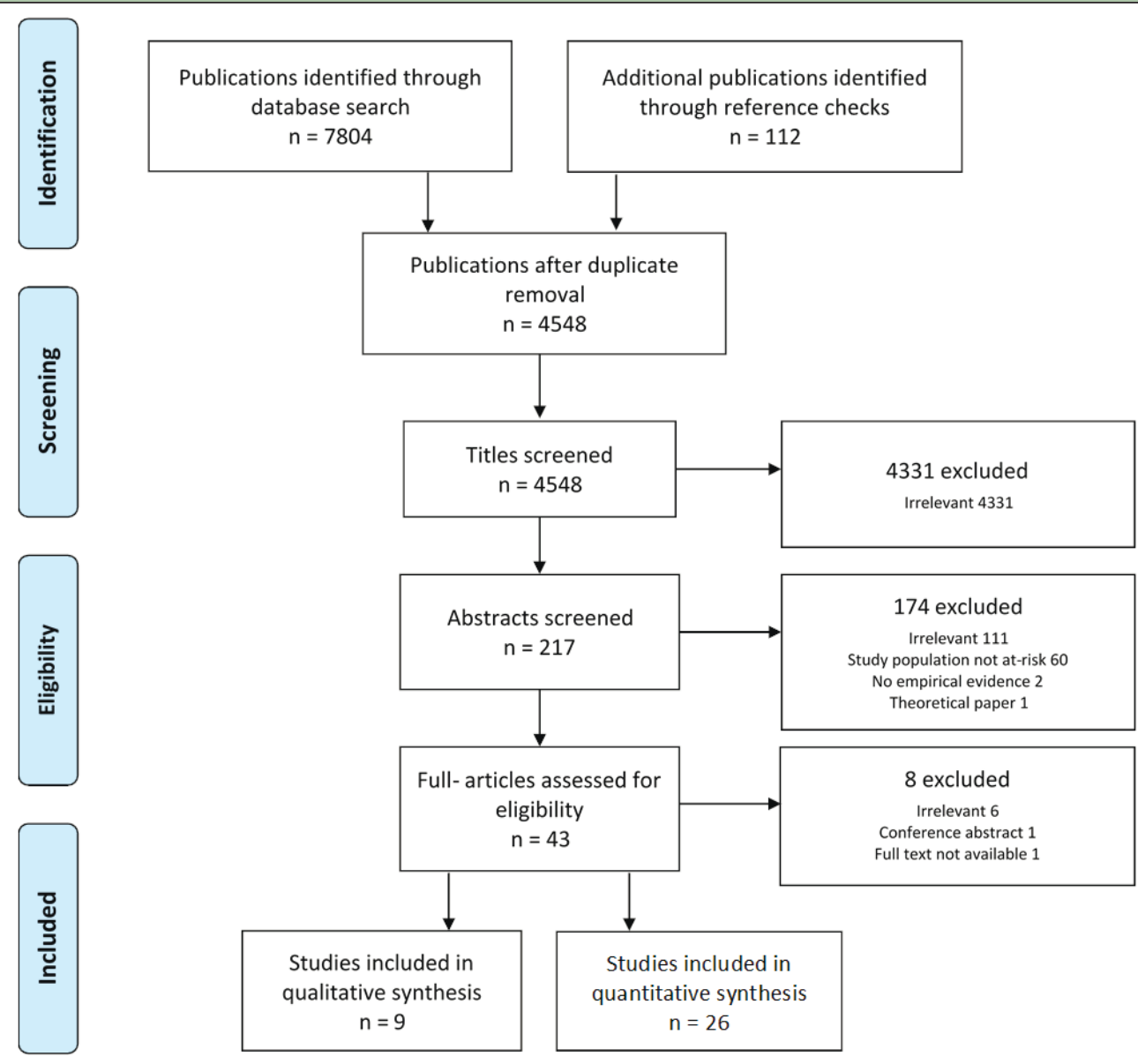

\section{Understanding}

\section{Understanding in cognitively impaired individuals}

Three studies investigated understanding, in terms of medical decision-making capacity in individuals with $\mathrm{MCI}$ and very mild dementia, using the validated Capacity to Consent to Treatment Instrument (CCTI) $(30,31)$. In addition, health numeracy, i.e. the ability to understand and use health-related numerical information, was studied in healthy and cognitively impaired individuals between the age of $50-95$ years (43). The studies showed that even MCI is associated 
with reduced competencies such as "understanding", "appreciation", and "reasoning". This is most likely related to impairment in basic cognitive domains including executive function and calculation abilities.

In contrast, two other studies that analyzed the impact of AD risk communication on MCI patients and their caregivers (26), as well as the effects of standardized counselling and risk disclosure of biomarker-based AD diagnosis (41), revealed that study participants generally comprehended the medical information.

\section{Understanding in cognitively unimpaired individuals}

Six studies investigated the ability to understand health and dementia-risk-related issues in healthy individuals with $\mathrm{AD}$-affected relatives $(16,22,27,33$, $34,50)$. At one-year post-disclosure only $48 \%$ of the participants were able to recall their lifetime risk estimate correctly, whereas $76 \%$ were able to report correctly the number of copies of the ("risk-enhancing") APOE4 allele they carried. Overall the studies indicated, that participants more likely remembered general information provided during the counselling and disclosure sessions, such as dichotomous information as being a risk gene carrier or not, than more complex information as specific genotypes or lifetime risk estimates. Furthermore, the studies indicated knowledge differences between ethnic groups, which were related to cultural factors and to inequalities in healthcare system access.

\section{Appraisal}

\section{Appraisal in cognitively impaired individuals}

Studies on appraisal of risk and AD-related health information in cognitively impaired individuals covered a wide range of aspects such as framing effects (44) and experiences as well as coping mechanisms when receiving diagnostic information $(40,46,48,49)$.

Framing effects refer to a bias that can be generated by the setting and the specific wording when communicating information. One study indicated that $\mathrm{MCI}$ and mild $\mathrm{AD}$ patients showed stronger proneness to framing effects as compared to controls. Such framing effects correlated inversely with performance in the domains of verbal and figural memory, attention span, executive functions and mental complex calculation (44).

One research group reported that MCI patients were highly interested in their brain amyloid status (49) and were aware of possible emotional effects after disclosure. Two studies in individuals with SCD and MCI reported effects of disclosure of amyloid status in real (40) and in hypothetical scenarios (48). Both concluded that psychological well-being was not negatively affected, when counselling and disclosure were performed at a specialized center and psychoeducational materials were offered.

Contrary, one study that used a qualitative approach to explore the experiences and coping mechanisms of MCI patients, indicated that the discussion of cognitive decline as a consequence of the amyloid status provoked negative emotions and reactions as well as problematic interaction with the family and the social environment (46). It was not investigated how these negative effects influence appraisal of health-related information, decision-making or health behavior, especially the decision to receive testing for $\mathrm{AD}$.

\section{Appraisal in cognitively unimpaired individuals}

The REVEAL-study group analyzed participants' perceptions of benefits and advantages (pros) and risks and limitations (cons) of genetic susceptibility testing (21). Initially, participants appraised the benefits as being more important than the limitations and risks. After performing genetic testing and disclosure of the test results, however, participants tended to have a less positive attitude and concerns increased. The result that benefits are initially prevailing in the decision-making process was confirmed by a Swedish study (50), where relatives of AD-patients stated, that, if available, they would go through a pre-symptomatic AD test. These results were confirmed in a Cuban study (45) that investigated the attitudes and knowledge of healthy relatives from a large Cuban family with monogenic EOAD patients.

The authors of the REVEAL-study found that risk disclosure of the APOE genotype had an impact on the individual participants' perceived risk of developing $\mathrm{AD}$ $(22,25,29)$, however, it did not have a clinically relevant psychological impact on the participants (28).

Two qualitative interview studies investigated experiences and coping mechanisms of cognitively healthy at-risk individuals for Alzheimer's dementia $(27,39)$. During the interviews some of the participants expressed feelings of helplessness and appraised their life and health as uncontrollable when reflecting about their risk of developing Alzheimer's dementia. Reported concerns referred to personal health and life perspective as well as to effects on other family members (27).

The REVEAL Qualitative Research Initiative (REVEALQRI) explored appraisal and coping preferences (39). The appraisal of potential emotional reactions to the APOEgenotype disclosure played a crucial role in the decision whether to proceed with genetic susceptibility testing or not. After APOE-genotype disclosure individuals developed problem-focused coping mechanisms such as financial planning and creating advance directives, but also search for more information and usage of biomedical tests to further clarify the individual risk. These findings were confirmed by quantitative research within the REVEAL-study group $(16,33)$. 


\section{Apply: Decision-Making}

\section{Decision-Making in cognitively impaired individuals}

Semi-structured interviews with MCI patients to capture the motivation to participate in real (49) and hypothetical (48) AD biomarker disclosure revealed that the most common reasons for choosing biomarker result disclosure were better understanding of their own brain condition and being able to make better decisions about future life planning. The need for a definite diagnosis preoccupied most of the participants. Possible negative social or legal consequences of the knowledge of one's biomarker status were not put forward. The authors found that MCI was associated with poorer financial and healthcare decision-making capacity, which in turn was related to worse global cognitive functioning (24).

\section{Decision-Making in cognitively unimpaired individuals}

One study examined the intentions for APOE genotyping within six hypothetical scenarios (32) and revealed that the overall interest in genetic testing was high and that greater interest in testing was associated with male gender and with scenarios describing high test accuracy, detailed information on risk and most importantly available treatment options. Seemingly, the appraisal of possible emotional reactions to the APOEgenotype disclosure are decisive whether to proceed with genetic susceptibility testing or not (39).

Intentions of changing health-related behavior were examined in a secondary analysis from data of the REVEAL-study $(20,37)$. The authors reported that APOE4-positive participants were more likely to think about insurance changes than APOE4-negative participants, especially regarding long-term care coverage.

\section{Apply: Effects on health behavior}

\section{Behavior change in cognitively impaired individuals}

One study in patients with mild AD reported that access to specialized health services, such as medical specialists or memory clinics, is mainly initiated by spouses or primary care physicians, but not by the patients themselves (47).

Within a clinical trial, individuals with SCD and a positive first-degree family history for AD completed amyloid positron-emission tomography (PET) scans and were engaged in a psychoeducational intervention with regard to AD (40) Participants who learned about their positive PET result reported that they had changed their lifestyle in terms of more physical exercise, healthier diet and planning ahead.

\section{Behavior change in cognitively unimpaired individuals}

The REVEAL-study group examined motivational aspects for participation in the APOE-disclosure study. The participants rated the following reasons as most important: contribution to research (94\%), arrangement of personal affairs (87\%), hope that effective treatment will be developed $(87 \%)$, arrangement of long-term care $(81 \%)$, preparation of family for the possibility of illness $(78 \%)$ and doing things sooner than planned $(75 \%)(35$, 36). Regarding participation in clinical trials with APOEgenotyping the study group identified higher household income, age below 60 years, Caucasian ethnicity and college graduate education status as predictors for seeking of genetic testing $(20,35,38)$.

Health behavior changes were examined in secondary analyses from data of the REVEAL-study. Five studies found an association between health behavior changes and APOE genotype $(19,20,23,35,42)$. APOE4positive participants were more likely to use additional medication or dietary supplements, mostly vitamins and/or botanical supplements and more likely to show changes in diet and exercise than APOE4-negative participants. The rates of health behavior change were similar in the APOE4-negative participants and in the control group.

\section{Discussion}

This systematic review shows that research on HL in at-risk individuals for Alzheimer's dementia is currently very limited. Existing results are fragmented and based on mostly small and often non-representative samples. The current literature shows that single aspects of HL have been addressed in research projects, but the concept of HL as a whole has not been embedded in study designs or outcome evaluations.

Nutbeam (8) has conceptualized three levels of $\mathrm{HL}$, the functional level, the interactive level and the critical level. Current research in subjects at risk of Alzheimer's dementia focuses on individual domains of $\mathrm{HL}$, including knowledge, attitudes, risk perception, lifestyle changes etc., which corresponds to the functional and the interactive level of HL (8). A core competence of functional literacy is sufficient cognitive capacity, which constitutes the basis for any further HL skill. This is of significant importance in HL related to $\mathrm{AD}$, since a fraction of the at-risk population (MCI) is cognitively impaired and others will become impaired in the course of the disease. At the same time, these individuals are faced with increasingly complex medical information due to rapid technical advances in the field early $\mathrm{AD}$ diagnosis, risk prediction and future treatments. This review highlights that reduced mental flexibility and amnestic and executive deficits severely impact on basic functional HL skills, such as 
comprehension $(24,30,31,43,48)$. The literature indicates that complex risk information is challenging for lay persons, and that information overload and the degree of information complexity may lead to misunderstanding and misperception of the presented information (16, $22,34)$. This could be indicated by either deficits in understanding risk-related information correctly, or an overestimation of benefits of genetic testing for AD, which may be due to several potential causes such as framing of the risk information, the need for certainty or the belief that there might be treatment for AD in the near future.

Individuals with higher levels of HL, such as the interactive level, are more likely to communicate their diagnosis and test results with family, friends, and their social environment. Discussing health-related topics such as positive and negative effects of early AD detection within the social environment can have a positive impact on psychological well-being and may result in a more reasonable and informed opinion in the individual but also in the social environment. Findings suggest that the role of the caregivers is of major importance, since their active presence in the disclosure session can positively contribute to the cognitive processing of information by the MCI patient and hence facilitate the comprehension of risk information (26). Interactive HL was only addressed by one study, where individuals discussed health-related topics with their social environment (17). The authors concluded that beliefs about AD risks and causes, genetic testing and development of treatment impact on the interaction pattern of the individuals with their social environment. Conversely, this process may influence the way medical information is appraised and may lead to a more reasonable and informed decision-making.

According to Nutbeam's HL concept, critical HL may deserve stronger attention in the context of AD. Critical $\mathrm{HL}$ is defined as a combination of advanced literacy skills and social skills, enabling an individual to critically analyze information and to use it to exert greater control over life events and situations. Findings from our review, in line with other research, show the strong wish for clarity concerning one's health risks and the need for clarification and a definite diagnosis, respectively (28, 40, 41, 49). Participants are willing to accept invasive diagnostics in order to attain certainty and control. In some cases, however, test outcomes may lead to the necessity of further assessments, and uncertainty may even increase. In addition, research on risk perception consistently suggests that people translate the statistical probability of a risk into a dichotomous information. When individuals are informed to be at risk, this may subjectively be equaled as being ill, including related negative psychosocial consequences. This indicates a potential misconception of risk states as opposed to disease diagnosis. Critical HL may therefore play an important role in the context $\mathrm{AD}$, since it may enable individuals to engage in decision-making in better accordance with their personal values and preferences.

Overall our findings highlight the paramount importance of adequate communication with persons at risk of $\mathrm{AD}$, which is sensitive to individual needs, skills, and preferences. Health care professionals are faced with unique complexities in the communication process when consulting MCI patients, as these patients have difficulties in handling and remembering abstract and complex risk information. Finally, HL skills are affected progressively in an individual with ongoing cognitive decline. In order to meet the individual requirements and needs of persons at risk for AD, there is a clear need to be flexible and responsive to patients' preferences for more basic or more detailed information. This is in line with the abovementioned conceptual framework underlying this review, i.e. that in order to promote individual HL, it needs to be embedded in a health literate environment (9).

A limitation of our review is, that most identified reports are based on data from the REVEAL-study group which provides a more comprehensive understanding of HL among offsprings of individuals with AD, who are considering genetic susceptibility testing for AD. However, this is a rare case since genetic susceptibility testing is not an established method for risk prediction in clinical practice. In fact, at present, APOE genotyping is not recommended in most guidelines, not even in the diagnostic process of patients with dementia (35). The current approaches of early AD detection and risk prediction of Alzheimer's dementia encompass biomarker-based diagnostics with cerebrospinal fluid (CSF) analysis or positron-emission tomography (PET). Regarding these technologies, studies on HL in at-risk groups are very limited. Furthermore, the findings of our review are limited in terms of evidence on HL in individuals at-risk for Alzheimer's dementia as an outcome and particularly regarding the assessment of validated tools for HL or its sub-dimensions.

\section{Conclusion}

This systematic review highlights the current research on HL of at-risk individuals for Alzheimer's dementia and reflects the need for more systematic research in this field. The results show that studies concentrate on single domains of $\mathrm{HL}$, but do not comprehensively investigate all steps required for reliable decisionmaking. A particular challenge in the field of $\mathrm{AD}$ is that cognitively impaired individuals are faced with a number of disease-immanent cognitive barriers which hinder them to apply HL skills the way cognitively "normal" individuals would do. Therefore, it will be of utmost importance to analyze their needs, and to provide essential information to facilitate their informed decisionmaking in the context of specific medical situations (e.g. biomarker-based predictive diagnosis of AD). Herein, it will be critical to adjust to patients' individual skills, 
needs, and preferences with respect to the level of detail of the information provided. One future direction for research is to identify the necessary requirements for an informed decision-making and for improved counselling of individuals at risk.

Contributors: Ayda Rostamzadeh and Julia Stapels conducted the literature review and were responsible for data analysis, data interpretation and writing the article. Mauro Seves and Theresa Haidl functioned as third reviewer to resolve discrepancies in the inclusion process. Anna Genske and Saskia Jünger developed the research methodology and assisted in writing the paper. All authors were engaged in the conceptualization, reviewed and commented on the manuscript. Frank Jessen and Christiane Woopen contributed to the conceptualization, writing, review of drafts of the article and obtained funding.

Funding: This project is funded by the Robert Bosch Foundation („Health literacy of persons at risk - from information to action (RisKomp)"; grant number 11.5.A402.0002.0) and by the Federal Ministry of Education and Research BMBF as part of the Network of European Funding for Neuroscience Research - ERA-NET NEURON („Ethical and Legal Framework for Predictive Diagnosis of Alzheimer's Disease: Quality of Life of Individuals at Risk and their Close Others" (PreDADQoL); funding number: 01GP1624). Both joint projects are conducted under the leadership of the Cologne Center for Ethics, Rights, Economics, and Social Sciences of Health (ceres). The sponsors did not have any influence on study initiation, conducting and reporting. The sponsors had no role in the design and conduct of the study; in the collection, analysis, and interpretation of data; in the preparation of the manuscript; or in the review or approval of the manuscript.

Acknowledgement: We are grateful to thank Christian Albus, Kristina Enders, Marc Hellstern, Samia Peltzer, Kerstin Rhiem, Stephan Ruhrmann and Rita Schmutzler for their valuable input and fruitful discussions during project meetings and scientific workshops. Moreover, we acknowledge Sophie Heseler for her support doing test searches that helped us refine our search strategy. Finally, we wish to thank Nicole Skoetz for her excellent methodological advice.

Declaration of conflicting interests: The authors declared no potential conflicts of this article.

Open Access: This article is distributed under the terms of the Creative Commons Attribution 4.0 International License (http://creativecommons.org/ licenses/by/4.0/), which permits use, duplication, adaptation, distribution and reproduction in any medium or format, as long as you give appropriate credit to the original author(s) and the source, provide a link to the Creative Commons license and indicate if changes were made.

\section{References}

1. Jack CR, Bennett DA, Blennow K, Carrillo MC, Dunn B, Haeberlein SB, et al NIA-AA Research Framework: Toward a biological definition of Alzheimer's disease. Alzheimer's and Dementia. 2018;14:535-62.

2. Albert MS, DeKosky ST, Dickson D, Dubois B, Feldman HH, Fox NC, et al. The diagnosis of mild cognitive impairment due to Alzheimer's disease: Recommendations from the National Institute on Aging-Alzheimer's Association workgroups on. Alzheimer's and Dementia. 2011;7:270-9.

3. Jessen F, Amariglio RE, Van Boxtel M, Breteler M, Ceccaldi M, Chetelat G, et al. A conceptual framework for research on subjective cognitive decline in preclinical Alzheimer's disease. Alzheimer's and Dementia. 2014;10:844-52.

4. Ngandu T, Lehtisalo J, Solomon A, Levälahti E, Ahtiluoto S, Antikainen R, et al. A 2 year multidomain intervention of diet, exercise, cognitive training, and vascular risk monitoring versus control to prevent cognitive decline in at-risk elderly people (FINGER): A randomised controlled trial. The Lancet. 2015;385:2255-63.

5. Barnes DE, Yaffe K. The projected effect of risk factor reduction on Alzheimer's disease prevalence. The Lancet Neurology. 2011;10:819-28.

6. Livingston G, Sommerlad A, Orgeta V, Costafreda SG, Huntley J, Ames D, et al. Dementia prevention, intervention, and care. The Lancet. 2017;390:2673734.

7. Sperling RA, Rentz DM, Johnson KA, Karlawish J, Donohue M, Salmon DP, et al. The A4 study: Stopping AD before symptoms begin? Science Translational Medicine. 2014;6.

8. Nutbeam D. Health literacy as a public health goal: a challenge for contemporary health education and communication strategies into the $21 \mathrm{st}$ century. Health Promotion International. 2000;15:259-67.

9. Sorensen K, Van den Broucke S, Fullam J, Doyle G, Pelikan JM, Slonska Z et al. Health literacy and public health: A systematic review and integration of definitions and models. BMC Public Health. 2012 Jan 25;12:80.

10. Australian Commission on Safety and Quality in Health Care. National statement on health literacy: taking action to improve safety and quality 2014 Available at http: / / safetyandquality.gov.au/wp-content/uploads / 2014/08/ Health-Literacy-National-Statement.pdf. Accessed 11th October 2017.
11. Moher D, Liberati A, Tetzlaff J, Altman DG, The PRISMA Group (2009). Preferred Reporting Items for Systematic Reviews and Meta-Analyses: The PRISMA Statement. BMJ 2009;339:b2535.

12. Thomas J, Harden A. Methods for the thematic synthesis of qualitative research in systematic reviews. BMC Medical Research Methodology. 2008;8.

13. Harden, Angela and James Thomas. «Mixed Methods and Systematic Reviews: Examples and Emerging Issues.» SAGE Handbook of Mixed Methods in Social \& Behavioral Research. Tashakkori, Abbas, editor, and Charles Teddlie, editor2nd ed Thousand Oaks: SAGE Publications, Inc., 2010, pp. 749-774.

14. Sandelowski M, Voils CI, Barroso J. Defining and Designing Mixed Research Synthesis Studies. Res Sch. 2006;13(1):29.

15. Hawker S, Payne S, Kerr C, Hardey M, Powell J. Appraising the evidence: Reviewing disparate data systematically. Qualitative Health Research. 2002;12:1284-99.

16. Akinleye I, Roberts JS, Royal CD, Linnenbringer E, Obisesan TO, Fasaye G-A GR. Differences between African American and white research volunteers in their attitudes, beliefs and knowledge regarding genetic testing for Alzheimer's disease. Journal of genetic counseling. 2011;20:650-659.

17. Ashida S, Koehly LM, Roberts JS, Chen CA, Hiraki S, Green RC. Disclosing the disclosure: Factors associated with communicating the results of genetic susceptibility testing for alzheimer's disease. Journal of Health Communication. 2009;14:768-84.

18. Ashida S, Koehly LM, Roberts JS, Chen CA, Hiraki S, Green RC. The role of disease perceptions and results sharing in psychological adaptation after genetic susceptibility testing: the REVEAL Study. European Journal of Human Genetics. 2010;18:1296-301.

19. Chao S, Roberts JS, Marteau TM, Silliman R, Cupples LA, Green RC. Health behavior changes after genetic risk assessment for Alzheimer disease: The REVEAL Study. Alzheimer disease and associated disorders. 22:94-7.

20. Christensen KD, Roberts J, Zikmund-Fisher BJ, Kardia S, McBride CM, Linnenbringer E, et al. Associations between self-referral and health behavior responses to genetic risk information. Genome Medicine. 2015;7:10.

21. Christensen KD, Roberts JS, Uhlmann WR GRcD. es to perceptions of the pros and cons of genetic susceptibility testing after APOE genotyping for Alzheimer disease risk. cs in medicine: official journal of the American College of Medical Genetics. 2011;193:118-25.

22. Eckert SL, Katzen H, Roberts JS, Barber M, Ravdin LD, Relkin NR, et al. Recall of disclosed apolipoprotein E genotype and lifetime risk estimate for Alzheimer's disease: the REVEAL Study. Genetics in medicine : official journal of the American College of Medical Genetics. 2006;8:746-51.

23. Fanshawe TR, Prevost a T, Roberts JS, Green RC, Marteau TM. Explaining Behavior Change after Genetic Testing: The Problem of Collinearity between Test Results and Risk Estimates Thomas. Genet Test. 2008;12:381-6.

24. Han SD, Boyle PA, James BD, Yu L, Bennett DA. Mild cognitive impairment is associated with poorer decision-making in community-based older persons. 2015;63:676-83.

25. Linnenbringer E, Roberts JS, Hiraki S, Adrienne L, Green RC. "I know what you told me, but this is what I think:" Perceived risk of Alzheimer disease among individuals who accurately recall their genetics-based risk estimate. Genet Med. 2010;12:219-27.

26. Guan Y, Roter DL, Erby LH, Wolff JL, Gitlin LN, Roberts JS, et al. Disclosing genetic risk of Alzheimer's disease to cognitively impaired patients and visit companions: Findings from the REVEAL Study. Patient Education and Counseling. 2017;100:927-35.

27. Wain KE, Uhlmann WR, Heidebrink J, Roberts JS. Living at risk: The sibling's perspective of early-onset alzheimer's disease. Journal of Genetic Counseling. 2009;18:239-51.

28. Green RC, Roberts JS, Cupples LA, Relkin NR, Whitehouse PJ, Brown T, et al. Disclosure of APOE genotype for risk of Alzheimer's disease. The New England journal of medicine. 2009;361:245-54.

29. Marteau TM, Roberts S, LaRusse S, Green RC. Predictive genetic testing for Alzheimer's disease: impact upon risk perception. Risk analysis : an official publication of the Society for Risk Analysis. 2005;25:397-404.

30. Okonkwo OC, Griffith HR, Belue K, Lanza S, Zamrini EY, Harrell LE, et al Medical decision-making capacity in mild cognitive impairment: a 3-year longitudinal study. Neurology. 2008;71:1474-1480 7p.

31. Okonkwo OC, Griffith HR, Belue K, Lanza S, Zamrini EY, Harrell LE, et al Medical decision-making capacity in patients with mild cognitive impairment. Neurology. 2007;69:1528-35.

32. Roberts JS. Anticipating Response to Predictive Genetic Testing for Alzheimer ' s Disease : A Survey of First-Degree Relatives. 2000;40:43-52.

33. Roberts IS, Connell CM. Illness representations among first-degree relatives of people with Alzheimer's disease. Alzheimer Disease and Associated Disorders. 2000;14:129-36.

34. Roberts, J. S., Chen, C. A., Uhlmann, W. R., Green, R. C., \& Group RS Effectiveness of a condensed protocol for disclosing APOE genotype and providing risk education for Alzheimer disease. Genetics in medicine: official journal of the American College of Medical Genetics. 2012;14:742-8.

35. Roberts JS, Cupples LA, Relkin NR, Whitehouse PJ, Green RC. Genetic Risk Assessment for Adult Children of People With Alzheimer's Disease: The Risk Evaluation and Education for Alzheimer's Disease (REVEAL) Study. Journal of Geriatric Psychiatry and Neurology. 2005;18:250-5.

36. Roberts JS, LaRusse S a, Katzen H, Whitehouse PJ, Barber M, Post SG, et al. Reasons for seeking genetic susceptibility testing among first-degree relatives of people with Alzheimer disease. Alzheimer disease and associated 
disorders. 2003;17:86-93.

37. Zick CD, Mathews CJ, Roberts JS, Cook-Deegan R, Pokorski RJ, Green RC. Genetic testing for Alzheimer's disease and its impact on insurance purchasing behavior. Health Affairs. 2005;24:483-90.

38. Roberts JS, Barber M, Brown TM, Cupples LA, Farrer LA, LaRusse SA, et al Who seeks genetic susceptibility testing for Alzheimer's disease? Findings from a multisite, randomized clinical trial. Genetics in Medicine. 2004;6:197203.

39. Gooding HC, Linnenbringer EL, Burack J, Roberts JS, Green RC, Biesecker BB. Genetic susceptibility testing for Alzheimer disease: Motivation to obtain information and control as precursors to coping with increased risk. Patient Education and Counseling. 2006;64:259-67.

40. Lim YY, Maruff P, Getter C, Snyder PJ. Disclosure of positron emission tomography amyloid imaging results: A preliminary study of safety and tolerability. Alzheimer's \& dementia : the journal of the Alzheimer's Association. 2016;12:454-8.

41. Lingler JH, Butters MA, Gentry AL, Hu L, Hunsaker AE, Klunk WE, et al. Development of a Standardized Approach to Disclosing Amyloid Imaging Research Results in Mild Cognitive Impairment. Journal of Alzheimer's disease : JAD. 2016;52:17-24

42. Vernarelli JA, Roberts JS, Hiraki S, Chen CA, Cupples LA, Green RC. Effect of Alzheimer disease genetic risk disclosure on dietary supplement use. American Journal of Clinical Nutrition. 2010;91:1402-7.

43. Delazer M, Kemmler G, Benke T. Health numeracy and cognitive decline in advanced age. Aging, Neuropsychology, and Cognition. 2013;20:639-59.
44. Zamarian L, Benke T, Buchler M, Wenter J, Delazer M. Information about medications may cause misunderstanding in older adults with cognitive impairment. Journal of the Neurological Sciences. 2010;298:46-51.

45. Marcheco-Teruel B, Fuentes-Smith E. Attitudes and knowledge about genetic testing before and after finding the disease-causing mutation among individuals at high risk for familial, early-onset Alzheimer's disease. Genetic testing and molecular biomarkers. 2009;13:121-5.

46. Banningh LJW, Vernooij-Dassen M, Rikkert MO, Teunisse JP. Mild cognitive impairment: Coping with an uncertain label. International Journal of Geriatric Psychiatry. 2008;23:148-54.

47. Bronner K, Perneczky R, McCabe R, Kurz A, Hamann J. Which medical and social decision topics are important after early diagnosis of Alzheimer's Disease from the perspectives of people with Alzheimer's Disease, spouses and professionals? BMC Research Notes. 2016;9:149.

48. Lawrence V, Pickett J, Murray J. Patient and carer views on participating in clinical trials for prodromal Alzheimer's disease and mild cognitive impairment. Int J Geriatr Psychiatry. 2014; 29: 22-31.

49. Vanderschaeghe G, Schaeverbeke J, Vandenberghe R, Dierickx K. Amnestic MCI Patients' Perspectives toward Disclosure of Amyloid PET Results in a Research Context. Neuroethics. 2017;10:281-97.

50. Axelman K, Lannfelt L, Almkvist O, Carlsson M. Life situation, coping and quality of life in people with high and low risk of developing Alzheimer's disease. Dementia and Geriatric Cognitive Disorders. 2003;16:220-8. 